\title{
Game Theoretic Framework for Power Control in InterCell Interference Coordination
}

\author{
Kinda Khawam*, Amine Adouane *, Samer Lahoud ${ }^{\dagger}$, Johanne Cohen ${ }^{\ddagger}$, Samir Tohme* \\ * University of Versailles - PRISM Laboratory, France \\ $\dagger$ University of Rennes I - IRISA, France \\ $\ddagger$ LRI, UPSud University of Paris South, France
}

\begin{abstract}
Inter-Cell Interference Coordination (ICIC) is commonly identified as a key radio resource management mechanism to enhance system performance of $4 \mathrm{G}$ networks. This paper addresses the problem of ICIC in the downlink of cellular OFDMA systems where the power level selection process of resource blocks (RB) is apprehended as a sub-modular game. The existence of Nash equilibriums (NE) for that type of games shows that stable power allocations can be reached by selfish Base Stations (BS). We put forward a semi distributed algorithm based on best response dynamics to attain the NEs of the modeled game. Based on local knowledge conveyed by the $\mathrm{X} 2$ interface in LTE (Long Term Evolution) networks [1], each BS will first select a pool of favorable RBs with low interference. Second, each BS will strive to fix the power level adequately on those selected RBs realizing performances comparable with the Max Power policy that uses full power on selected RBs while achieving substantial power economy. Finally, we compare the obtained results to an optimal global solution to quantify the efficiency loss of the distributed game approach. It turns out that even though the distributed game results are sub-optimal, the low degree of system complexity and the inherent adaptability make the decentralized approach promising especially for dynamic scenarios.
\end{abstract}

\section{INTRODUCTION}

Orthogonal Frequency Division Multiplexing (OFDM) is widely accepted as the access scheme for $4 \mathrm{G}$ wireless standards thanks to its numerous merits. Because of the OFDM orthogonality feature, the intra-cell interference is mostly mitigated and can be ignored. However, for the inter-cell interference (ICI), when the frequency resource is universally reused in each cell of the system, users with bad channel quality, in particular cell-edge users, can suffer from high interference caused by neighboring cells. Proposing efficient ways to hinder inter-cell interference is paramount ([2], [3], [4], [5]). In this work, we propose to reduce ICI through efficient Power Control. Power control does not only reduce the impact of interfering signals by lowering their power level (signals usually belonging to cell-center users), but it can increase the power level on resource blocks that suffer of bad radio conditions (usually RB allocated for cell-edge users). Therefore, it is considered as a method for Inter-Cell Interference Coordination (ICIC) [6].

Downlink power allocation in LTE systems can vary from cell to cell and can be, in each cell, device specific. Several works have proposed and studied Power Control algorithms ISBN 978-3-901882-58-6 (c) 2014 IFIP in OFDM systems. In [7], authors put forward a distributed power control algorithm where each cell aims independently at minimizing its own power consumption under users rate constraints. Despite the absence of cooperation between the different cells, the power allocation scheme takes into account the interference due to the usage of the same resources in neighboring cells. Power control is done iteratively according to the proposed Bit Allocation Algorithm until the allocated power levels remain invariant after two consecutive iterations. In [8], a hybrid algorithm combines adaptive modulation along with power control (i.e., the power level is increased when the order of the modulation scheme decreases and vice versa). It permits using high order modulation schemes at low Signal to Noise Ratio values without degrading the system performance. In [9], an Adaptive Power Control (APC) is proposed to reduce inter-cell interference as excessively high transmission power for center-cell users will degrade the performances of edge-cell users. Hence, power level for cell-center users is lowered at the profit cell-edge users. A Fair SINR strategy is sought for where power is distributed among users in a way to obtain the same Signal-to-Interference and Noise Ratio (SINR) at the receiver. In [10], two power control algorithms are proposed to automatically create soft fractional frequency reuse (FFR) patterns in OFDMA based systems. The goal of the proposed algorithms is to adjust the transmit powers of the different RBs by systematically pursuing maximization of the overall network utility. The first algorithm is semi-distributed as it relies on gradient information exchanged periodically by neighboring cells, whereas the second one is fully distributed relying on a non-trivial heuristic. The work in ([11],[12]) builds upon the work in [10]. The work in [11] extends the proposed algorithms for multi antenna OFDM systems with space division multiple access. The work in [12] differs mainly in the granularity of power control where the power is set on a per-beam basis.

In general, central entities performing the task of interference coordination with global knowledge should be avoided. Therefore, our work belongs to the category of decentralized ICIC that is sought for. Resorting to non-cooperative game theory is quite suitable to model the way BSs compete in a distributed manner for shared resources. Devising an optimal power level selection scheme depends on the existence of Nash equilibriums for the present game ensuring that the distributed 
coordination process will iteratively reach a stable state. In this paper, we prove that the model at hand is a sub-modular game (see [15], [14]). Such games have always a Nash Equilibrium and it can be attained using a greedy best response type algorithm (called algorithm I in both references). The result of the devised coordination process in each cell will be the power tuning on the least interfered RBs.

The rest of the paper is organized as follows. The system model is given in Section II. In Section III, the framework of the RB selection and power control schemes is described. The power level selection scheme is presented as a non-cooperative sub-modular game in Section IV where a semi distributed learning algorithm based on best response dynamics is presented in subsection IV-C. Simulations results are portrayed in Section V. The optimal centralized approach is given in VI as a benchmark to evaluate the price of anarchy resulting from a decentralized approach. Conclusion is given in Section VII.

\section{The System Model}

We consider a cellular network comprising $M=\{1, \ldots, m\}$ hexagonal BSs. Each BS can communicate with its 6 neighboring BSs (using the X2 interface). We focus on the downlink scenario in this paper. OFDMA is used as the multiple access scheme. The time and frequency radio resources are grouped into time-frequency Resource Blocks (RBs). RB is the smallest radio resource block that can be scheduled to a mobile user. Each RB consists of $N_{s}$ OFDM symbols in the time dimension and $N_{f}$ sub-carriers in the frequency dimension (in LTE, $N_{s}=7$ and $N_{f}=12$ ). The total number of RBs is denoted by $n$ and $N=\{1, \ldots, n\}$ represents the set of RBs. Both BSs and mobile users have a single antenna each.

\section{A. Data Rate on the Downlink}

Hereafter, we compute the SINR perceived on the downlink to derive the data rate per user on a given RB. The SINR observed on RB $k$ alloted to user $u$ in $\mathrm{BS} i$ can be expressed as follows:

$$
S I N R_{i, k, u}=\frac{P_{0} \cdot G_{i} \cdot x_{i, k} \cdot\left(\frac{1}{d_{u, i}^{i}}\right)^{\beta}}{P_{0} \cdot \sum_{\substack{j \in M \\ j \neq i}}, G_{j} \cdot x_{j, k} \cdot\left(\frac{1}{d_{u, j}^{i}}\right)^{\beta}+P_{N}}
$$

where $P_{0}$ represents the maximal transmitted power per RB, $P_{N}$ represents the thermal noise power per RB, $G_{i}$ the antenna gain of BS $i, d_{u, j}^{i}$ is the distance between BS $j$ and user $u$ served by BS $i$ and $\beta$ is the path-loss factor varying between 2 and 6 . Finally, $x_{i, k}$ is the discrete variable that represents a fraction of the maximal transmitted power $P_{0}$. Hence, $x_{i, k}^{l}=$ $P_{0} \cdot x_{i, k}$ is one of the possible $N_{l}$ power levels in BS $i$ on RB $k$ alloted to user $u$. It varies between $x_{i, k}^{l}=0.0$ where $\mathrm{RB} k$ is not selected by BS $i$ and $x_{i, k}^{l}=P_{0}$ where full power is transmitted on that RB. However, our power control starts after the selection of a pool of RBs in any BS $i$ as will be explained in the next section. Hence, $x_{i, k}^{l} \neq 0.0$.

We denote by $D_{i, k, u}$ the data rate achieved by user $u$ on RB $k$ in $\mathrm{BS} i$ given by what follows [16]:

$$
D_{i, k, u}=\frac{W}{E_{b} / N_{0}} \cdot S I N R_{i, k, u},
$$

where $W$ is the bandwidth per RB. Given a target error probability, it is necessary that $E_{b} / N_{0} \geq \gamma$, for some threshold $\gamma$ which is user specific. The assumption of a constant $E_{b} / N_{0}$ target is generally valid as long as the same type of modulation is used for all data rates [17].

In any $\mathrm{BS} i$, the cell will be logically divided into $N_{Z}$ concentric discs of radii $R_{z}, z=1, \ldots, N_{Z}$, and the area between two adjacent circles of radii $R_{z-1}$ and $R_{z}$ is called zone $z, z=1, \ldots, N_{z}$. We assume that mobile users belonging to the same zone $z$ have the same radio conditions leading to the same $\gamma_{z}$ and to the same mean rate per zone $D_{i, k, z}$ on RB $k$ according to what follows:

$$
\begin{aligned}
D_{i, k, z} & =\frac{\frac{W}{\gamma_{z}} \int_{R_{z-1}}^{R_{z}} \frac{2 r d r}{R_{c e l l}^{2} r^{\beta}} \cdot G_{i} \cdot P_{0} \cdot x_{i, k}}{P_{0} \cdot \sum_{\substack{j \in M \\
j \neq i}} G_{j} \cdot x_{j, k} \cdot \frac{1}{\left(d_{z, j}^{i}\right)^{\beta}}+P_{N}} \\
& =\frac{\left(\frac{W}{\gamma_{z}} \frac{R_{z}^{2-\beta}-R_{z-1}^{2-\beta}}{R_{c e l l}^{2}}\right) \cdot G_{i} \cdot P_{0} \cdot x_{i, k}}{P_{0} \cdot \sum_{\substack{j \in M \\
j \neq i}}, x_{j, k} \cdot \frac{G_{j}}{\left(\delta_{i, j}^{z} \cdot R_{c e l l}\right)^{\beta}}+P_{N}}
\end{aligned}
$$

where $R_{\text {cell }}$ is the cell radius. As for interference, we adopt an optimistic simplification where we only consider the impact of BS $j$ on BS $i$ by replacing $d_{u, j}^{i}$ by $d_{z, j}^{i}=\delta_{i, j}^{z} \cdot R_{c e l l}$ which is the distance between BS $i$ and BS $j$ (the value of $\delta_{i, j}^{z}$ depends on how far is $\mathrm{BS} j$ from zone $z$ of $\mathrm{BS} i$ ).

\section{B. Cost Function}

Our goal is to put forward an efficient power control algorithm that enables each BS to set appropriately the amount of transmitted power in order to satisfy serviced mobile users. Satisfaction for a mobile user is defined here as the minimization of the transfer time it perceives subsequent to a given power level selection. However, the cost function adopted is not only an image of transfer time but reflects also global welfare by inflecting on BSs a punishment proportional to the power level adopted. The goal is to select power levels that minimize transfer time while steering away BSs from excessively high power allocations that exacerbate interference.

We denote by $T_{i, k, z}$ the amount of time necessary to send a data unit through RB $k$ in BS $i$ for users in zone $z$. In fact, the delay needed to transmit a bit for a given user is the inverse of the data rate perceived by this user:

$$
T_{i, k, z}=\frac{I_{i, k, z}}{x_{i, k}}
$$

where $I_{i, k, z}$ is given by:

$$
I_{i, k, z}=\frac{\sum_{\substack{j \in M \\ j \neq i}} x_{j, k} \cdot H_{i, j}^{z}+P_{N}}{H_{i, z}}
$$

where $H_{i, j}^{z}=\frac{P_{0} G_{j}}{\left(\delta_{i, j}^{z} \cdot R_{c e l l}\right)^{\beta}}$ captures distance-dependent attenuation of power between BS $j$ and zone $z$ of BS $i$ and $H_{i, z}=\left(\frac{W}{\gamma_{z}} \frac{R_{z}^{2-\beta}-R_{z-1}^{2-\beta}}{R_{c e l l}^{2}}\right) \cdot P_{0} G_{i}$ captures distance-dependent 
attenuation of power inside zone $z$ of BS $i$.

We denote by $R B_{z}^{i}$ the pool of RBs used by BS $i$ users in zone $z$. BS $i$ will pay an amount $\alpha_{z}$ per power unit transmitted on a given RB $k \in R B_{z}^{i}$. This power unitary cost can decrease with the zone index to further protect users that are far away from the antenna; or it can increase to favor cell-center users in order to enhance overall performances.

Accordingly, the goal of the power control scheme proposed in this paper is to minimize the following cost function in BS $i$ for $\mathrm{RB} k$ alloted to a user in zone $z$ :

$c_{i, k, z}=\kappa \cdot T_{i, k, z}+\alpha_{z} \cdot P_{0} \cdot x_{i, k}$, If $\mathrm{RB} k$ is used in zone $z$

$=0$, If $\mathrm{RB} k$ is not used in zone $z$

where $\kappa$ is a normalization factor.

\section{PROPOSED ICIC SCHEME}

In this paper, we propose that each BS aggregates information about transmit power levels in adjacent cells and sets accordingly the pool of RBs to be used in any zone. The RNTP (Relative Narrow-band Transmit Power) indicator, received from neighboring BSs every 200 TTI (Transmit Time Interval) through the X2 interface, advertises on which RBs a neighboring BS will use a power level that surpasses a given threshold. In any $\mathrm{BS} i$, we associate with every RB $k$ a variable $a_{i, k}$. This variable indicates the number of neighboring BSs that advertised the use of that same $\mathrm{RB} k$ with full power via the RNTP indicators $\left(0 \leq a_{i, k} \leq 6\right)$. Thus, every BS $i$ updates its variables $a_{i, k}$ approximately every 200 TTI and makes use of those variables to update the pool of selected RBs per zone as described in algorithm 1 where $n_{z}^{i}$ is the number of mobile users in zone $z$ of BS $i$. Recall that $R B_{z}^{i}$ is the pool of RBs reserved to users in zone $z$.

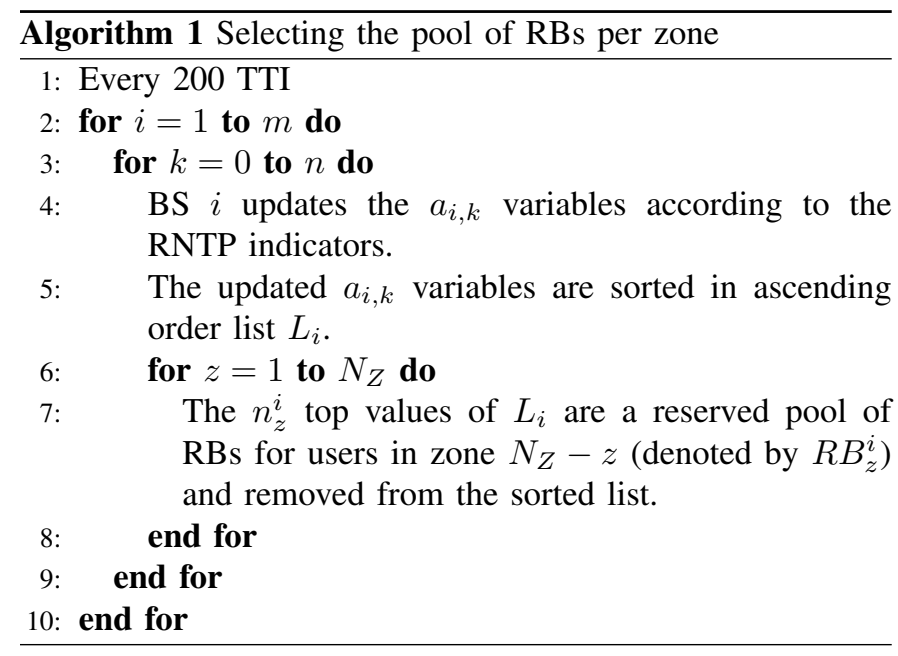

The idea behind the algorithm is to reserve the pool of least interfered RBs to users who are the furthest away from the BS (the zones with the highest index). After selecting $R B_{z}^{i}$, every $\mathrm{BS} i$ proceeds to implementing the power control distributed algorithm described in the following section and which is the focal point of the paper. We assume that the scheduler has already assigned users in a given zone $z$ to $\mathrm{RBs}$ in $R B_{z}^{i}$. Afterwards, our proposed power control scheme endeavors to find the optimal power levels on those allocated RBs. After convergence of the latter, each BS $i$ will obtain the optimal power level $P_{i, k, z}^{*}$ to be assigned to $\mathrm{RB} k$ in zone $z$.

\section{Non-CoOperative Game For Power Control}

Non-Cooperative game theory models the interactions between players competing for a common resource. Hence, it is well adapted to ICIC modeling. Here, BSs are the decision makers or players of the game. We define a multi-player game $\mathcal{G}$ between the $m$ BSs. The BSs are assumed to make their decisions without knowing the decisions of each other.

The formulation of this non-cooperative game $G=$ $\langle M, S, C\rangle$ can be described as follows:

- A finite set of players $M=(1, \ldots, m)$ and a finite set of RBs $N=(1, \ldots, n)$.

- For each BS $i$, the space of strategies $S$ is formed by the Cartesian product of each set of strategies $S_{i}=S_{i, 1} \times$ $\ldots \times S_{i, n}$, where $n$ is the total number of RBs. An action of a BS $i$ is the amount of power $x_{i, k}$ sent on RB $k$. If $\mathrm{RB} k \in R B_{z}^{i}$ then $S_{i, k}=\left\{x_{i, k}^{0}, \ldots, x_{i, k}^{N_{l}-1}\right\}$ where $x_{i, k}^{j}$ is a fraction of $P_{0}$, else $S_{i, k}=\emptyset$. The strategy chosen by BS $i$ is then $X_{i}=\left(x_{i, 1}, \ldots, x_{i, n}\right)$. A strategy profile $X=\left(X_{1}, \ldots, X_{m}\right)$ specifies the strategies of all players and $S=S_{1} \times \ldots \times S_{m}$ is the set of all strategies.

- A set of cost functions $C=\left(C_{1}(X), C_{2}(X), \ldots, C_{m}(X)\right)$ that quantify players' costs for a given strategy profile $X$ where $C_{i}=\left(c_{i, 1, z}, c_{i, 2, z}, \ldots, c_{i, n, z}\right)$ is the cost of BS $i$ with $c_{i, k, z}$ the cost of using RB $k$ in zone $z$ as given in (5). As the frequencies alloted to different RBs are orthogonal, minimization of cost $c_{i, k, z}$ on $\mathrm{RB} k$ is done independently of other RBs. Hence, we denote by $x_{-i, k}$ the strategies played by all BSs on RB $k$ except BS $i$.

\section{A. The Nash Equilibrium}

In a non-cooperative game, an efficient solution is obtained when all players adhere to a Nash Equilibrium (NE). A NE is a profile of strategies in which no player will profit from deviating its strategy unilaterally. Hence, it is a strategy profile where each player's strategy is an optimal response to the other players' strategies.

$$
\begin{aligned}
& c_{i, k, z}\left(x_{i, k}, x_{-i, k}\right) \leq c_{i, k, z}\left(x_{i, k}^{\prime}, x_{-i, k}\right), \\
& \forall i \in M, \forall z \in N_{Z}, \forall k \in R B_{z}^{i}, \forall x_{i, k}^{\prime} \in S_{i, k}
\end{aligned}
$$

We turn to sub-modularity theory to show existence of Nash equilibriums.

\section{B. Sub-modular Game}

S-modularity was introduced into the game theory literature by Topkis [15] in 1979. S-modular games are of particular interest since they have Nash equilibriums, and there exists an upper and a lower bound on Nash strategies of each user 
[18]. Furthermore, these equilibriums can be attained by using a greedy best response type algorithm ([15], [14]).

Definition 4.1: Consider a game $G=\langle M, S, C\rangle$ with strategy spaces $S_{i} \subset \mathbb{R}^{m}$ for all $i \in M$ and for all $z \in N_{Z}, k \in R B_{z}^{i}$. $G$ is sub-modular if for each $i$ and $k, S_{i, k}$ is a sublattice ${ }^{1}$ of $\mathbb{R}^{m}$, and $c_{i, k, z}\left(x_{i, k}, x_{-i, k}\right)$ is sub-modular in $x_{i, k}$.

Definition 4.2: The cost function $c_{i, k, z}$ is sub-modular iff for all $x, y \in S_{i, k}$,

$c_{i, k, z}(\min (x, y))+c_{i, k, z}(\max (x, y)) \leq c_{i, k, z}(x)+c_{i, k, z}(y)$

Proposition 4.3: The cost function $c_{i, k, z}$ is sub-modular for every BS $i$ and zone $z$ and every selected RB $k \in R B_{z}^{i}$.

The proof of this proposition can be found in the appendix of the paper A. Since $S_{i, k}$ is a single dimensional finite set, $S_{i, k}$ is a compact sublattice of $\mathbb{R}$. And as the cost function $c_{i, k, z}$ is sub-modular for every BS $i$ on every selected RB $k \in R B_{z}^{i}$, our game is indeed sub-modular.

\section{Attaining the Nash Equilibrium}

1) The Best Response Dynamics: The best response strategy of player $i$ is the one that minimizes its cost given other players strategies. A best response dynamics scheme consists of a sequence of rounds, each player $i$ chooses the best response to the other players strategies in the previous round. In the first round, the choice of each player is the best response based on its arbitrary belief about what the other players will choose. In some games, the sequence of strategies generated by best response dynamics converges to a NE, regardless of the players initial strategies. S-modular games are part of those games.

To reach the NE, [13] proposes the following greedy best response algorithm built on an algorithm called algorithm I in [14], [15]: there are $T$ infinite increasing sequences $T_{t}^{i}$ for $t \in T$ and $i=1, \ldots, m$. Player $i$ uses at time $T_{k}^{i}$ the best response policy (a feasible one) to the policies used by all other players just before $T_{k}^{i}$. This scheme includes in particular parallel updates (when $T_{t}^{i}$ does not depend on $t$ ). Once this user updates its strategy, the strategies of one or more other users need not be feasible anymore. In [13], proof is given for the following two results:

- If each player $i$ either initially uses its lowest or largest policy in $S_{i}$, then the iterative algorithm converges monotonically to an equilibrium (that may depend on the initial state).

- If we start with a feasible policy, then the sequence of best responses monotonically converges to an equilibrium: it monotonically decreases in all components in the case of cost minimizing in a sub-modular game.

BS $i$ strives to find, for the pool of selected RBs in any zone $z$, the following optimal power level:

$$
P_{i, k, z}^{*}=P_{0} \cdot \arg \min _{x_{i, k}} c_{i, k, z}\left(x_{i, k}, x_{-i, k}\right),
$$

${ }^{1} A$ is a sublattice of $\mathbb{R}^{m}$ if $a \in A$ and $a^{\prime} \in A$ imply $a \wedge a^{\prime} \in A$ and $a \vee a^{\prime} \in A$ for $P_{0} \cdot x_{i, k} \in\left\{x_{i, k}^{0}, \ldots, x_{i, k}^{N_{l}-1}\right\}$.

By definition, $P_{i, k, z}^{*}$ is a best response of BS $i$ to the other BSs strategies on RB $k$ in zone $z$.

2) Distributed Learning of $N E$ : In a real environment, a best response type algorithm as the one proposed in ([14], [15]) cannot be practically applied as every BS $i$ needs to know the policy of all other BSs $x_{-i, k}$ on every used RB $k$ which necessitates expensive signaling and hinders the benefits of an efficient power control scheme. Fortunately, we can easily render our algorithm distributed by making use of signaling information already present in the downlink of an LTE system. In fact, $x_{-i, k}$ (or equivalently $x_{j, k} \forall j \neq i$ ) only intervene in the total interference $I_{i, k, z}$ endured on RB $k$ in zone $z$ of BS $i$ according to equation (4). In practice, mobile users measure the channel quality based on pilots, i.e., Cell Specific Reference Signals (CRS) that are spread across the whole band independently of the individual users allocation. In particular, interference can be easily inferred through the CQI (Channel Quality Indicator) sent every TTI by the mobile users to which RB $k$ is attributed. However, the BSs should update their transmission powers on selected RBs sequentially in a predefined round robin fashion that need to be set once and for all.

We present in Algorithm 2 the pseudo-code of our distributed power control scheme under best response dynamics deemed DBR:

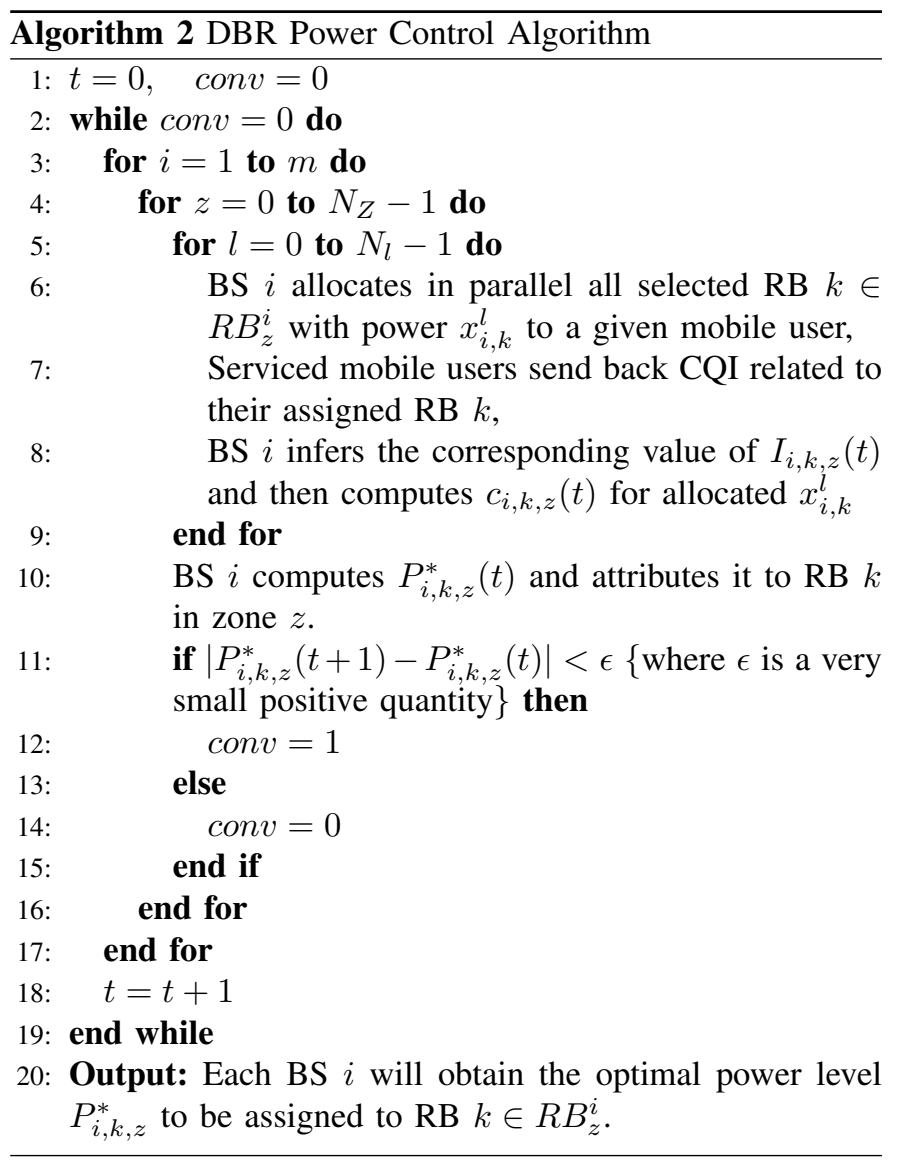




\section{Simulation Results}

We consider a bandwidth of $10 \mathrm{MHz}$ with $50 \mathrm{RBs}$ along with the following parameters listed in the 3GPP technical specifications TS 36.942: the mean antenna gain in urban zones is $12 \mathrm{dBi}$. As for noise, we consider the following: user noise figure 7.0 dB, thermal noise $-104.5 \mathrm{dBm}$ which gives a receiver noise floor of $P_{N}=-97.5 \mathrm{dBm}$. We consider 9 hexagonal cells where each cell is surrounded with 6 other cells. The distance between two neighboring BS is $1 \mathrm{Km}$. Maximum transmission power is $43 \mathrm{dBm}$ (according to TS 36.814) on the downlink. We set $P_{0}=10$ Watts and $x_{i, k}$ for any BS $i$ on RB $k$ belongs to $\{0.1,0.2,0.35,0.5,0.6,0.7,0.85,1.0\}$. Various power unitary costs $\left(\alpha_{z}\right)$ were tested and for each scenario 400 simulations were run where in each cell a random number of users is chosen in every zone corresponding to a snapshot of the network state. Performances are compared against Max Power policy where full power $P_{0}$ is used on all RBs and against Random policy where power levels are set at random. For every simulation, 100 runs of Random policy were made. Further, for each simulation instance, the same pool of RBs per zone is given for the three policies DBR, Max Power policy and Random policy. Hence, results only assess the impact of power control. Only two zones are taken into account: zone 1 which stands for cell-center users located at a distance smaller than $R_{0}=0.5 \mathrm{Km}$ and zone 2 stands for celledge users located at a distance ranging between $R_{0}=0.5 \mathrm{Km}$ and $R_{1}=R_{\text {cell }}=1 \mathrm{Km}$. We assume that for cell-center users, 64-QAM modulation is used while for cell-edge users, 16QAM modulation is used.

In Figure 1, we depict the total transfer time per zone $T_{z}=\sum_{i \in M} \sum_{k \in R B_{z}^{i}} T_{i, k, z}$ for cell-center and cell-edge users as a function of various power unitary costs $\left(\alpha_{1} ; \alpha_{2}\right)$ for DBR and Max Power Policy. In most scenarios, we aimed at favoring cell-edge users by lowering the power unitary cost in comparison to that of cell-center users. We notice as expected that the improvement in one zone as compared to the Max Power policy is obtained at the expense of the other zone. This fact is highlighted in the lowest sub-figure where the relative deviation $D e v_{T}=\frac{T_{z}^{D B R}-T_{z}^{M a x P o w e r}}{T_{z B R}^{D}} * 100$ is displayed. Further, we see that the improvement in one zone does not strictly depend on how low its power unitary cost is but on how low it is relatively to the other zone: despite the fact that no power unitary cost is inflected on cell-edge users in scenario $(1 ; 0)$, the total transfer time is greater than that of scenarios $(2 ; 0.2)$ or $(4 ; 0.2)$.

We denote by $T$ the global transfer time given by:

$$
T=\sum_{i \in M} \sum_{z=1}^{2} \sum_{k \in R B_{z}^{i}} T_{i, k, z}
$$

In Figure 2, we depict the global transfer time $T$ as a function of power unitary cost for DBR, Max Power policy and Random policy. Except for $(0.2 ; 3)$ and $(4 ; 0.2)$ where there is a large discrepancy between the power unitary cost of one zone in comparison with the other, performances of DBR and Max Power policy are equivalent for all other scenarios. However,

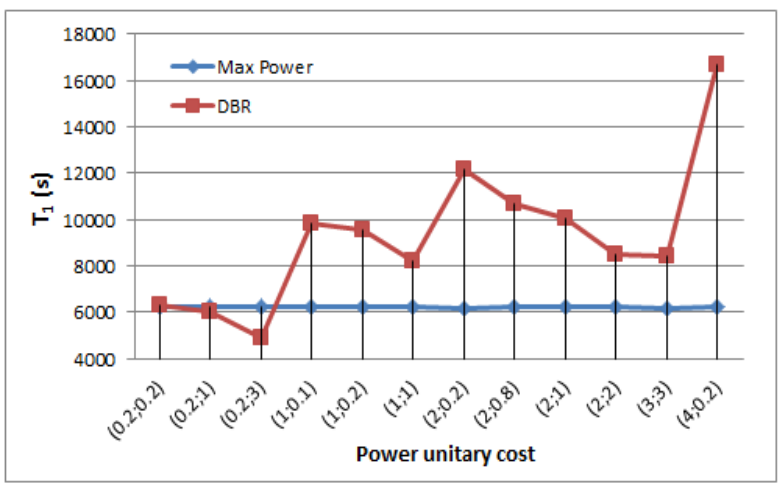

(a) Cell-center Users

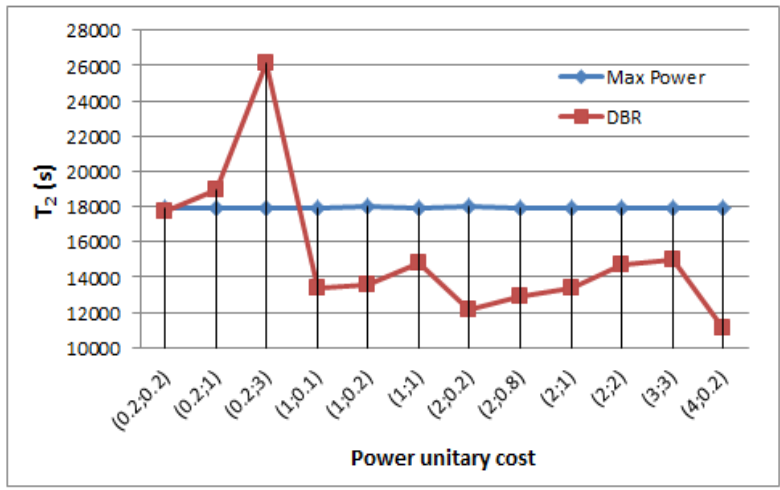

(b) Cell-edge Users

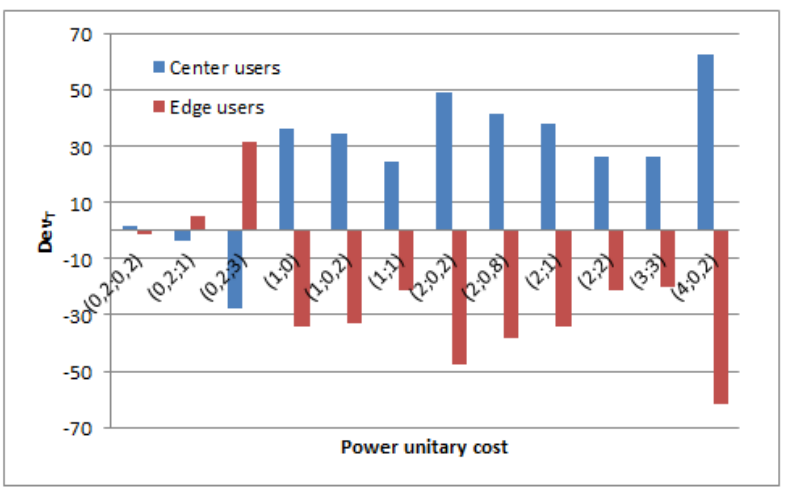

(c) Relative Deviation

Figure 1. Transfer Time per zone as a function of power unitary cost for DBR vs. Max Power Policy

DBR permits a considerable power economy in comparison with Max Power policy as we can see in Figure 3 where the relative deviation, denoted $D e v_{P}$, between the total power consumed in DBR and total power consumed in the Max Power policy is displayed as a function of power unitary cost. We can clearly see that the highest the power unitary cost is, the highest the power economy and vice versa. The best performances are reached when the same (high) power unitary cost is assigned for both zones in scenarios $(2 ; 2)$ and $(3 ; 3)$ where power economy vary from $70 \%$ till $80 \%$ while the total transfer time is slightly lower than that in the Max 
Power policy. As for the Random policy, we can see that performances are mediocre.

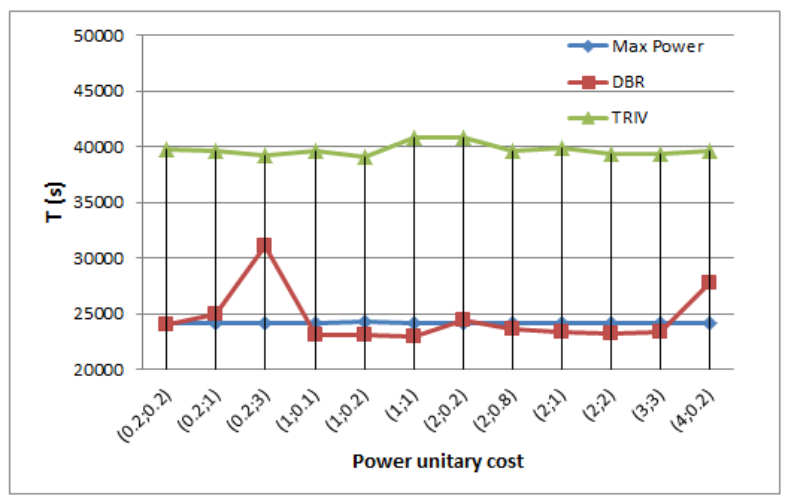

Figure 2. Total Transfer Time as a function of power unitary cost for DBR vs. Max Power Policy and Trivial Policy

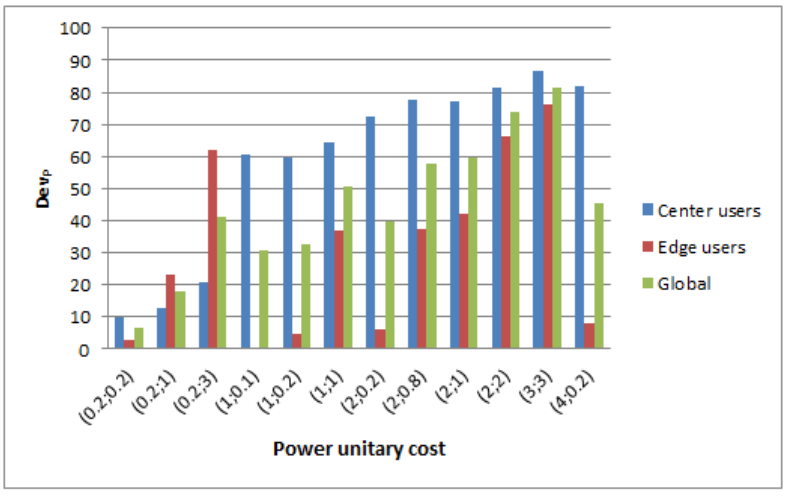

Figure 3. Power Economy

In Figure 4, we report the mean convergence time as a function of power unitary cost. We note that DBR attains NE faster than 110 TTI and hence before the exchange of new RNTP messages (sent every 200 TTI) which is a very appealing characteristic.

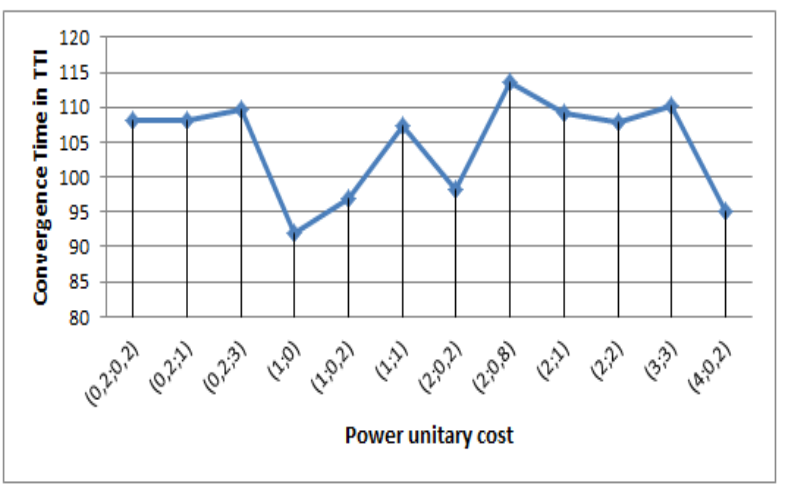

Figure 4. Convergence Time

\section{THE PRICE OF ANARCHY}

In this section, we quantify the loss in efficiency suffered when a distributed scheme is adopted rather than a centralized optimization which is commonly known as the price of anarchy.

\section{A. Optimal Centralized Approach}

Unlike the distributed approach where precedence is given to the interests of each individual BS, power control may be performed in a way that favors the overall system performance. We do so by introducing a centralized approach, where a central controller assigns the power levels of each BS in order to minimize the total network cost. This total network cost is given by the sum of the costs for all BSs. Note that due to the orthogonality of the frequencies alloted to different RBs, minimization of the total network cost is done independently for each RB. Hence, the centralized approach consists in solving a set of optimization problems given by $\mathcal{P}_{k}$ for each $k \in R B_{z}^{i}$ and denoted in the following:

$$
\begin{array}{r}
\left(\mathcal{P}_{k}\right): \operatorname{minimize} C\left(x_{i, k}\right)=\sum_{i \in M} \sum_{z \in N_{z}} c_{i, k, z} \\
x_{i, k}=\{0.1,0.2,0.35,0.5,0.6,0.7,0.85,1.0\}, \forall i \in M .
\end{array}
$$

$\mathcal{P}_{k}$ are integer non-linear optimization problems that are typically hard to solve [22]. Therefore, we start by relaxing the integrality constraints on $x_{i, k}$, i.e., we assume that $0<$ $x_{i, k} \leq 1$ for all $i \in M$ and $k \in R B_{z}^{i}$. After relaxing the integrality constraints, the obtained optimization problems $\widehat{\mathcal{P}_{k}}$ are non-linear convex problems given hereafter:

$$
\begin{array}{r}
\left(\widehat{\mathcal{P}_{k}}\right): \operatorname{minimize} C\left(x_{i, k}\right)=\sum_{i \in M} \sum_{z \in N_{z}} c_{i, k, z} \\
0<x_{i, k} \leq 1, \forall i \in M .
\end{array}
$$

The convexity property of the $\widehat{\mathcal{P}_{k}}$ optimization problems enables to efficiently solve large instances using for example an interior point method. However, the challenge remains in recovering the integer solution of the original problems $\mathcal{P}_{k}$. In what follows, we consider a basic approach that rounds towards infinity the solution of the relaxed problems $\widehat{\mathcal{P}_{k}}$. Let us denote by $\left\{x_{i, k}^{*}, i \in M\right\}$ the optimal solution vector of the original problem $\mathcal{P}_{k}$ for each $k \in N, \mathcal{P}_{k},\left\{\widehat{x_{i, k}}, i \in M\right\}$ the optimal solution vector of the relaxed problem $\widehat{\mathcal{P}_{k}}$, and $\left\{\left\lceil\widehat{x_{i, k}}\right\rceil, i \in M\right\}$ the rounded vector, we can easily write:

$$
C\left(\widehat{x_{i, k}}\right) \leq C\left(x_{i, k}^{*}\right) \leq C\left(\left\lceil\widehat{x_{i, k}}\right\rceil\right) .
$$

Although we do not directly compute the optimal integral solution, equation (12) provides a bounding interval and thus gives us insights on the price of the anarchy. In the following, we compare the performances of the distributed approach with the rounded solution (an upper bound on the solution obtained by the original problem) thus we get an upper bound on the price of anarchy. 


\section{B. Simulation Results}

In Figure 5, we illustrate the global transfer time $T$ (given by (9)) necessary to send a data unit for all users as a function of the system load for DBR, the Optimal policy, and the Max Power policy. We see that the performances of DBR and the Optimal policy are equivalent and that they slightly outperforms the Max Power approach that systematically resorts to full power which degrades the SINR and in turn increases the transfer time.

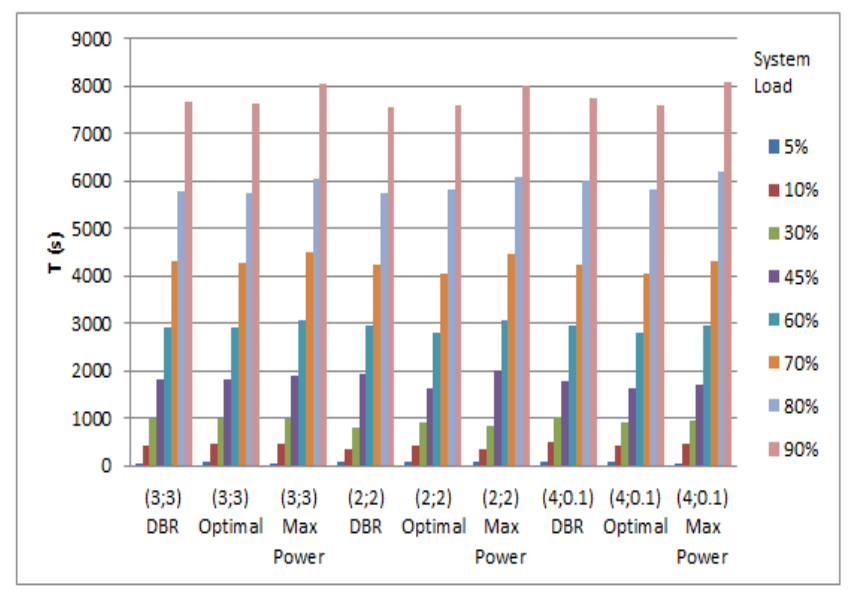

Figure 5. Global Transfer Time as a function of power unitary cost for DBR, Max Power and Optimal policies

However, the power economy made in the optimal approach as compared to DBR tempers its benefits as we can see from Figure 6, where the relative deviation between the total power in DBR (respectively in the Optimal policy) and the Max Power policy is displayed as a function of power unitary cost. It is obvious that the optimal policy saves up much more power than the decentralized approach even in high load whereas the power economy in DBR withers slowly as load increases. Nevertheless, the slight discrepancy between the global transfer time in DBR and the Optimal policy which the primary goal sought for and the low degree of system complexity of the decentralized approach makes it still an attractive solution.

\section{CONCLUSION}

In this paper, the power levels are astutely set as part of the LTE Inter Cell Interference coordination process. We proposed a semi distributed algorithm based on best response dynamics to reach NEs in a time coherent with the RNTP signaling time. The DBR algorithm takes into consideration both income of transfer time and punishment of power wastage. Numerical simulations assessed the good performances of the proposed approach in comparison with a policy that services active users with full power. More importantly, considerable power saving can be realized. Furthermore, the performance of the proposed algorithm was analyzed by simulations with respect to the efficiency loss compared to a centralized optimization. It turns out, although the power economy realized by the optimal

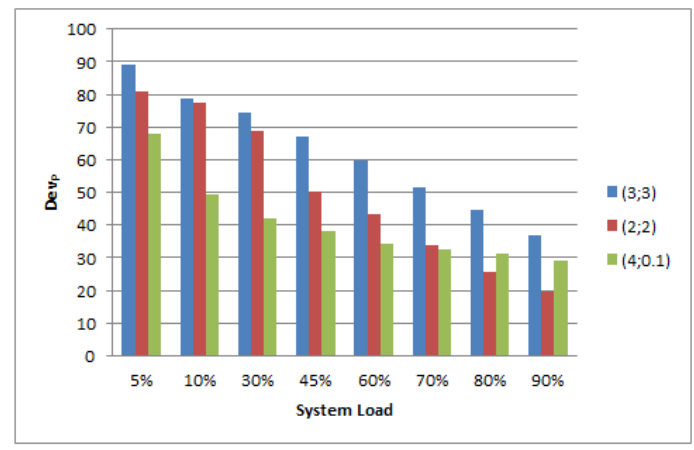

(a) DBR Policy

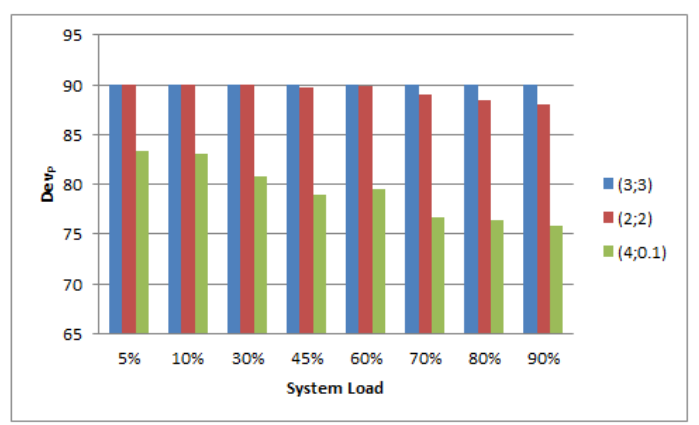

(b) Optimal Policy

Figure 6. Power Economy as a function of power unitary cost for DBR and Optimal policies

centralized approach is much higher, the discrepancy in terms of transfer time is negligible. Hence, as efficient power control can be realized based on local information only, the efficiency loss is largely counterbalanced by a high degree of flexibility and relatively low convergence time.

\section{APPENDIX}

\section{A. Proof of sub-modularity}

Our goal is to prove that the cost function $c_{i, k, z}$ is submodular for every BS $i$ and zone $z$ and every selected RB $k \in R B_{z}^{i}$.

Proof: We begin by defining the set $A_{1}$ such that $A_{1}=\left\{x_{j, k}, y_{j, k} \in S_{j, k} \mid x_{j, k}<y_{j, k}, j \neq i\right\}$ and $A_{2}$ such that $A_{2}=\left\{x_{j, k}, y_{j, k} \in S_{j, k} \mid x_{j, k}>y_{j, k}, j \neq i\right\}$. Accordingly, the inequality in (8) gives the following for $x=\left(x_{j, k}, j=1, \ldots, m\right)$ and $y=\left(y_{j, k}, j=1, \ldots, m\right)$ where $x_{j, k}, y_{j, k} \in S_{j, k}$ :

$$
\begin{aligned}
& \kappa \cdot \sum_{j \neq i} \frac{\min \left(x_{j, k}, y_{j, k}\right) H_{i, j}^{z}+P_{N}}{H_{i, z} \min \left(x_{i, k}, y_{i, k}\right)}+\alpha_{z} P_{0} \min \left(x_{i, k}, y_{i, k}\right) \\
& +\kappa \cdot \sum_{j \neq i} \frac{\max \left(x_{j, k}, y_{j, k}\right) H_{i, j}^{z}+P_{N}}{H_{i, z} \max \left(x_{i, k}, y_{i, k}\right)}+\alpha_{z} P_{0} \max \left(x_{i, k}, y_{i, k}\right) \\
& \leq \kappa \cdot \sum_{j \neq i} \frac{x_{j, k} H_{i, j}^{z}+P_{N}}{H_{i, z} x_{i, k}}+\kappa \cdot \sum_{j \neq i} \frac{y_{j, k} H_{i, j}^{z}+P_{N}}{H_{i, z} y_{i, k}} \\
& +P_{0} \alpha_{z}\left(x_{i, k}+y_{i, k}\right)
\end{aligned}
$$


We notice in (13) that $P_{0} \alpha_{z}\left(\min \left(x_{i, k}, y_{i, k}\right)+\right.$ $\left.\max \left(x_{i, k}, y_{i, k}\right)\right)=P_{0} \alpha_{z}\left(x_{i, k}+y_{i, k}\right)$ and $P_{N} \cdot(m-$ $1)\left(\frac{1}{\min \left(x_{i, k}, y_{i, k}\right)}+\frac{1}{\max \left(x_{i, k}, y_{i, k}\right)}\right)=P_{N} \cdot(m-1)\left(\frac{1}{x_{i, k}}+\frac{1}{y_{i, k}}\right)$ where $m$ is the total number of BSs. Thus, inequality (13) simplifies to:

$$
\begin{aligned}
& \sum_{j \neq i} \frac{\min \left(x_{j, k}, y_{j, k}\right) H_{i, j}^{z}}{\min \left(x_{i, k}, y_{i, k}\right)}+\sum_{j \neq i} \frac{\max \left(x_{j, k}, y_{j, k}\right) H_{i, j}^{z}}{\max \left(x_{i, k}, y_{i, k}\right)} \\
& \leq \sum_{j \neq i} \frac{x_{j, k} H_{i, j}^{z}}{x_{i, k}}+\sum_{j \neq i} \frac{y_{j, k} H_{i, j}^{z}}{y_{i, k}}
\end{aligned}
$$

Using the sets $A_{1}$ and $A_{2}$, inequality (14) is re-written as follows:

$$
\begin{aligned}
& \sum_{j \in A_{1}} \frac{x_{j, k} H_{i, j}^{z}}{\min \left(x_{i, k}, y_{i, k}\right)}+\sum_{j \in A_{2}} \frac{y_{j, k} H_{i, j}^{z}}{\min \left(x_{i, k}, y_{i, k}\right)} \\
& \sum_{j \in A_{1}} \frac{y_{j, k} H_{i, j}^{z}}{\max \left(x_{i, k}, y_{i, k}\right)}+\sum_{j \in A_{2}} \frac{x_{j, k} H_{i, j}^{z}}{\max \left(x_{i, k}, y_{i, k}\right)} \\
& \leq \sum_{j \in A_{1}} \frac{x_{j, k} H_{i, j}^{z}}{x_{i, k}}+\sum_{j \in A_{2}} \frac{x_{j, k} H_{i, j}^{z}}{x_{i, k}} \\
& +\sum_{j \in A_{1}} \frac{y_{j, k} H_{i, j}^{z}}{y_{i, k}}+\sum_{j \in A_{2}} \frac{y_{j, k} H_{i, j}^{z}}{y_{i, k}}
\end{aligned}
$$

Here, before going further, we need to distinguish two cases:

- Case 1: $x_{i, k}<y_{i, k}$. In this case, inequality (15) gives the following:

$$
\begin{aligned}
& \sum_{j \in A_{1}} \frac{x_{j, k} H_{i, j}^{z}}{x_{i, k}}+\sum_{j \in A_{2}} \frac{y_{j, k} H_{i, j}^{z}}{x_{i, k}} \\
& \sum_{j \in A_{1}} \frac{y_{j, k} H_{i, j}^{z}}{y_{i, k}}+\sum_{j \in A_{2}} \frac{x_{j, k} H_{i, j}^{z}}{y_{i, k}} \\
& \leq \sum_{j \in A_{1}} \frac{x_{j, k} H_{i, j}^{z}}{x_{i, k}}+\sum_{j \in A_{2}} \frac{x_{j, k} H_{i, j}^{z}}{x_{i, k}} \\
& +\sum_{j \in A_{1}} \frac{y_{j, k} H_{i, j}^{z}}{y_{i, k}}+\sum_{j \in A_{2}} \frac{y_{j, k} H_{i, j}^{z}}{y_{i, k}}
\end{aligned}
$$

After some simplifications, inequality (16) gives what follows:

$$
\sum_{j \in A_{2}}\left(x_{j, k}-y_{j, k}\right) H_{i, j}^{z}\left(\frac{1}{y_{i, k}}-\frac{1}{x_{i, k}}\right)<0
$$

The latter inequality is obviously true as $x_{j, k}>$ $y_{j, k}, \forall j \neq i$ in $A_{1}$ and $x_{i, k}<y_{i, k}$.

- Case 2: $x_{i, k}>y_{i, k}$. Similarly to case 1, inequality (15) simplifies to:

$$
\sum_{j \in A_{1}}\left(y_{j, k}-x_{j, k}\right) H_{i, j}^{z}\left(\frac{1}{y_{i, k}}-\frac{1}{x_{i, k}}\right)>0
$$

The latter inequality is obviously true as $y_{j, k}>$ $x_{j, k}, \forall j \neq i$ in $A_{2}$ and $x_{i, k}>y_{i, k}$.

\section{REFERENCES}

[1] 3GPP TS 36.211 V8.3.0, Evolved Universal Terrestrial Radio Access; Physical Channels and Modulation, June 2008

[2] R. Y. Chang, Z.Tao, J. Zhang and C. -C. J. Kuo, A Graph Approach to Dynamic Fractional Frequency Reuse (FFR) in Multi-Cell OFDMA Networks, IEEE International Conference on Communications, pp.16, June 2009

[3] T. Quek, Z. Lei and S. Sun, Adaptive interference coordination in multi-cell OFDMA systems, IEEE 20th International Symposium on Personal, Indoor and Mobile Radio Communications, pp.2380-2384, September 2009.

[4] M. Rahman, H. Yanikomeroglu and W. Wong, Interference Avoidance with Dynamic Inter-Cell Coordination for Downlink LTE System, Wireless Communications and Networking Conference, pp.1-6, April 2009.

[5] S. G. Kim, K. Cho, D. Yoon, Y-J. Ko and J. K. Kwon, Performance Analysis of Downlink Inter Cell Interference Coordination in the LTEAdvanced System, Fourth International Conference on Digital Telecommunications, pp.30-33, July 2009.

[6] G. Boudreau. J. Panicker, N. Guo, R. Chang, N. Wang and S. Vrzic, Interference coordination and cancellation for $4 \mathrm{G}$ networks, Communications Magazine, IEEE , vol.47, no.4, pp.74-81, April 2009

[7] P. Bisaglia, S. Pupolin, D. Veronesi, and M. Gobbi, Resource Allocation and Power Control in a TDD OFDM-Based System for 4G Cellular Networks, in IEEE 63rd Vehicular Technology Conf., Melbourne, 2006

[8] I. I. M. Al-Kebsi, M. Ismail, The Impact of Modulation Adaptation and Power Control on PAPR Clipping Technique in OFDM of 4G Systems, in 6th Nat. Conf. Telecommunication Technologies and 2nd Malaysia Conf. Photonics, Putrajaya, 2008.

[9] R. Schoenen and F. Qin, Adaptive Power Control for 4G OFDMA Systems on Frequency Selective Fading Channels, in 5th Int. Conf. Wireless Communications, Networking and Mobile Computing, Beijing, 2009.

[10] A. L. Stolyar and H. Viswanathan, Self-Organizing Dynamic Fractional Frequency Reuse for Best-Effort Traffic Through Distributed Inter-Cell Coordination, in IEEE INFOCOM, Rio de Janeiro, 2009.

[11] G. Wunder, M. Kasparick, A. Stolyar, and H. Viswanathan, SelfOrganizing Distributed Inter-Cell Beam Coordination in Cellular Networks with Best Effort Traffic, in WiOpt 2010.

[12] M. Kasparick and G. Wunder, Autonomous Distributed Power Control Algorithms for Interference Mitigation in Multi-Antenna Cellular Networks, in 11th European Wireless Conf. Sustainable Wireless Technologies, Vienna, 2011.

[13] Altman E. and Altman Z., S-Modular Games and Power Control in wireless Networks, IEEE Transactions on Automatic Control, vol. 45, no 5, 2003.

[14] Yao, S-modular games, with queueing applications, Queueing systems, vol. 21, no 3-4 (239 p.), pp. 449-475, 1995.

[15] Topkis, Equilibrium Points in Non-Zero Sum n-Person Sub-modular Games, SIAM J. Control Optim. Volume 17, Iss. 6, 773-787, 1979.

[16] Bonald T., Proutiere A., Wireless downlink data channels: user performance and cell dimensioning, MobiCom '03: Proceedings of the 9th annual international conference on Mobile computing and networking.

[17] S-L. Kim, Z. Rosberg, J. Zander, Combined Power Control and Transmission Rate Selection In Cellular Networks, in: Proc.of IEEE VTC Fall, 1999.

[18] Martin J. Osborne and Ariel Rubinstein, A course in game theory, MIT Press, 1994; Chinese translation published by China Social Sciences Publishing House, 2000.

[19] 3GPP TS 36.211 V8.9.0 Physical channels and modulation (Release 8), 2009-12

[20] 3GPP TS 36.212 V8.8.0 Multiplexing and channel coding (Release 8), 2009-12

[21] 3GPP TS 36.213 V8.8.0 Physical layer procedures (Release 8), 200909

[22] Hemmecke, R., Kppe, M., Lee, J., and Weismantel, R. Nonlinear integer programming. In 50 Years of Integer Programming 1958-2008 (pp. 561-618). Springer Berlin Heidelberg. 